\title{
Effects of high-frequency, high- intensity transcutaneous electrical nerve stimulation versus intravenous opioids for pain relief after gynecologic laparoscopic surgery: a randomized controlled study
}

\author{
Birgitta Platon ${ }^{1}$, Clas Mannheimer ${ }^{2}$, and Paulin Andréll ${ }^{3}$ \\ ${ }^{1}$ Department of Anaesthesiology and Intensive Care Medicine, Sahlgrenska University Hospital, ${ }^{2}$ Multidisciplinary \\ Pain Center Kungälv Hospital Kungälv and Institute of Medicine, Sahlgrenska Academy at the University of \\ Gothenburg, Sahlgrenska University Hospital, ${ }^{3}$ Department of Molecular and Clinical Medicine/Multidisciplinary \\ Pain Center, Institute of Medicine, Sahlgrenska Academy at the University of Gothenburg, Sahlgrenska University \\ Hospital, Gothenburg, Sweden
}

\begin{abstract}
Background: The aim of the study was to compare the pain relieving effect and the time spent in the recovery unit after treatment with high frequency, high-intensity transcutaneous electrical nerve stimulation (TENS) or intravenous (IV) opioids after gynecologic laparoscopic surgery.

Methods: All patients who postoperatively reported visual analogue scale (VAS) pain score $\geq 3$ were consecutively included in the study. The TENS treatment was given with a stimulus intensity between 40-60 mA during 1 minute, repeated once if insufficient pain relief. In the opioid group, a maximum dose of $10 \mathrm{mg}$ morphine was given IV. If the patient reported insufficient pain relief (VAS $\geq 3$ ) on the assigned treatment, the patient crossed over to the other treatment group.

Results: Ninety-three women were randomized to TENS $(n=47)$ or IV opioids $(n=46)$. Both groups reported significant pain relief at leave from the recovery unit (TENS group: VAS 5.4 to 1.0, $\mathrm{P}<0.001$; IV opioid group: VAS 5.2 to 1.1, $\mathrm{P}<0.001$ ) with no differences between the groups. When only responders, i.e. patients with VAS $<3$ after assigned treatment, were compared the TENS responders spent significantly shorter time in the recovery unit $(90$ vs. 122 minutes, $\mathrm{P}=$ 0.008) compared to the responders in the opioid group.

Conclusions: TENS and IV opioids are both effective treatments for pain relief after gynecologic laparoscopic surgery. TENS seems to be preferable for first choice of treatment as the treatment is associated with shorter time spent in recovery unit if the patient responds to the treatment.
\end{abstract}

Keywords: Gynecologic; Laparoscopy; Opioid; Pain; Transcutaneous electrical nerve stimulation.

\footnotetext{
Corresponding author: Birgitta Platon, R.N.

Department of Anaesthesiology and Intensive Care Medicine, Sahlgrenska University Hospital, Östra, SE-416 85 Gothenburg, Sweden

Tel: 463134344 46, Fax: 46313435737

Email: birgitta.platon@vgregion.se

ORCID: https://orcid.org/0000-0002-9992-9578
}

Received: February 17, 2017. Revised: October 25, 2017 (1st); December 1, 2017 (2nd). Accepted: December 13, 2017.

Korean J Anesthesiol 2018 April 71(2): 149-156

https://doi.org/10.4097/kjae.2018.71.2.149

(c) This is an open-access article distributed under the terms of the Creative Commons Attribution Non-Commercial License (http://creativecommons.org/ licenses/by-nc/4.0/), which permits unrestricted non-commercial use, distribution, and reproduction in any medium, provided the original work is properly cited. 


\section{Introduction}

Laparoscopic surgery has become the standard approach for a large number of gynecologic procedures. The frequency of laparoscopy varies widely across the globe. In the United States, approximately 350,000 tubal ligations and 200,000 laparoscopically assisted vaginal hysterectomies are performed annually. Whereas, in the United Kingdom, approximately 250,000 gynecologic laparoscopic surgeries are performed annually [1].

At our center, approximately 200 laparoscopic operations are performed annually for the following indications: sterilization, diagnosis, cyst enucleation, and salpingo-oophorectomy.

Pain is often a problem after gynecologic laparoscopic surgery, even if the postoperative pain is less significant when compared to that after a similar major laparotomy surgery [2]. Conventional treatment with intravenous (IV) opioids often offers satisfactory pain relief. However, opioids have negative side effects, such as sedation, nausea, and respiratory depression [3]. Some of these side effects require monitoring of the patient, resulting in longer duration of stay in the recovery unit after surgery.

Transcutaneous electrical nerve stimulation (TENS) has been used to provide pain relief in various postoperative pain conditions such as post-thoracotomy pain, pain following spinal surgery, after abdominal surgery, laparoscopic tubal ligation, pain after cholecystectomy, as a complement for pain relief after pancreatic resection, and colon surgery [4-10].

The intensity of stimulation seems to be of importance for pain relief as studies indicate that a strong, non-painful intensity seems to be more efficacious [11]. High-frequency, high-intensity (40-60 mA) TENS has been used in pain treatment after surgical abortions, resulting in effective pain relief and a shorter stay in the recovery unit [12]. However, to our knowledge, the data regarding the effect of high-frequency, high-intensity TENS for postoperative pain treatment after gynecologic laparoscopic surgery is scarce. Our hypothesis was that patients receiving TENS for pain relief after undergoing gynecologic laparoscopic surgery would spend a shorter amount of time in the recovery unit than those treated with IV opioids. The objective of the current prospective, randomized study was to compare the duration of stay in the recovery unit after surgery and to assess the pain-relieving effect of high-frequency, high-intensity TENS and conventional treatment with IV opioids in patients undergoing gynecologic laparoscopic surgery.

\section{Materials and Methods}

\section{Patients}

The study is in accordance with the Helsinki Declaration of 1975, as revised in 2000. The Regional Ethics Review Board in Gothenburg, Sweden, approved the study (Dnr 026-11). All participants received written and verbal information about the study before giving their written consent to participate in the study. The study is registered in a national register (VGR Dnr 221131, http://www.fou.nu/is/vgr/project/221131).

Over 13 months, patients admitted to the operating theater at Sahlgrenska University Hospital/Östra for gynecologic laparoscopic surgery were assessed for eligibility (Table 1). Every patient who reported a postoperative pain score of $\geq 3$ on the visual analog scale (VAS) in the recovery unit after laparoscopic surgery was randomized using the closed-envelope technique (Fig. 1). Both the patient and investigator were aware of the randomized allocation. Based on our clinical experience, blinding was not feasible since high-intensity TENS was needed to achieve optimal pain relief. Hence, it was impossible to blind the patient to the treatment allocation. The exclusion criteria were unwillingness to participate in the study, alcohol or drug addiction, neurological disease with impaired sensitivity, use of a pacemaker or implantable cardioverter defibrillator, dependence on an interpreter, or age less than 18 years.

\section{Procedures related to surgery}

The standard procedure prior to gynecologic laparoscopic surgery was applied and the patients received an in-dwelling IV peripheral catheter.

All patients received IV or oral paracetamol and/or non-steroidal anti-inflammatory drugs (NSAID), pre-, intra-, and postoperatively, according to clinical routine during the hospital stay (every $8 \mathrm{~h}$ ). The patients also received their ordinary pain medi-

Table 1. Patient Demographics and Indications for Gynecologic Laparoscopic Surgery in the TENS Treatment Group and the IV Opioid Treatment Group

\begin{tabular}{lcc}
\hline & $\begin{array}{c}\text { TENS group } \\
(\mathrm{n}=47)\end{array}$ & $\begin{array}{c}\text { IV opioid group } \\
(\mathrm{n}=46)\end{array}$ \\
\hline Baseline health characteristics & & \\
Age & $43(13)$ & $42(11)$ \\
Previous abdominal surgery & $17(36 \%)$ & $16(35 \%)$ \\
Long-term pain & $16(34 \%)$ & $18(39 \%)$ \\
Analgesic use* & $17(36 \%)$ & $21(46 \%)$ \\
Indications for gynecologic laparoscopic surgery & \\
Sterilization & $9(19 \%)$ & $9(20)$ \\
Diagnosis & $2(4 \%)$ & $14(30 \%)$ \\
Cyst enucleation & $18(38 \%)$ & $11(24 \%)$ \\
Salpingo-oophorectomy & $14(30 \%)$ & $7(15 \%)$ \\
Adhesion lysis and others & $4(9 \%)$ & $5(11 \%)$ \\
\hline
\end{tabular}

Data are presented as number of patients (\%) or mean (SD). TENS: transcutaneous electrical nerve stimulation, IV: intravenous, NSAID: non-steroidal anti-inflammatory drugs. *Analgesics include NSAIDs, paracetamol, and opioids, including tramadol and codeine. 


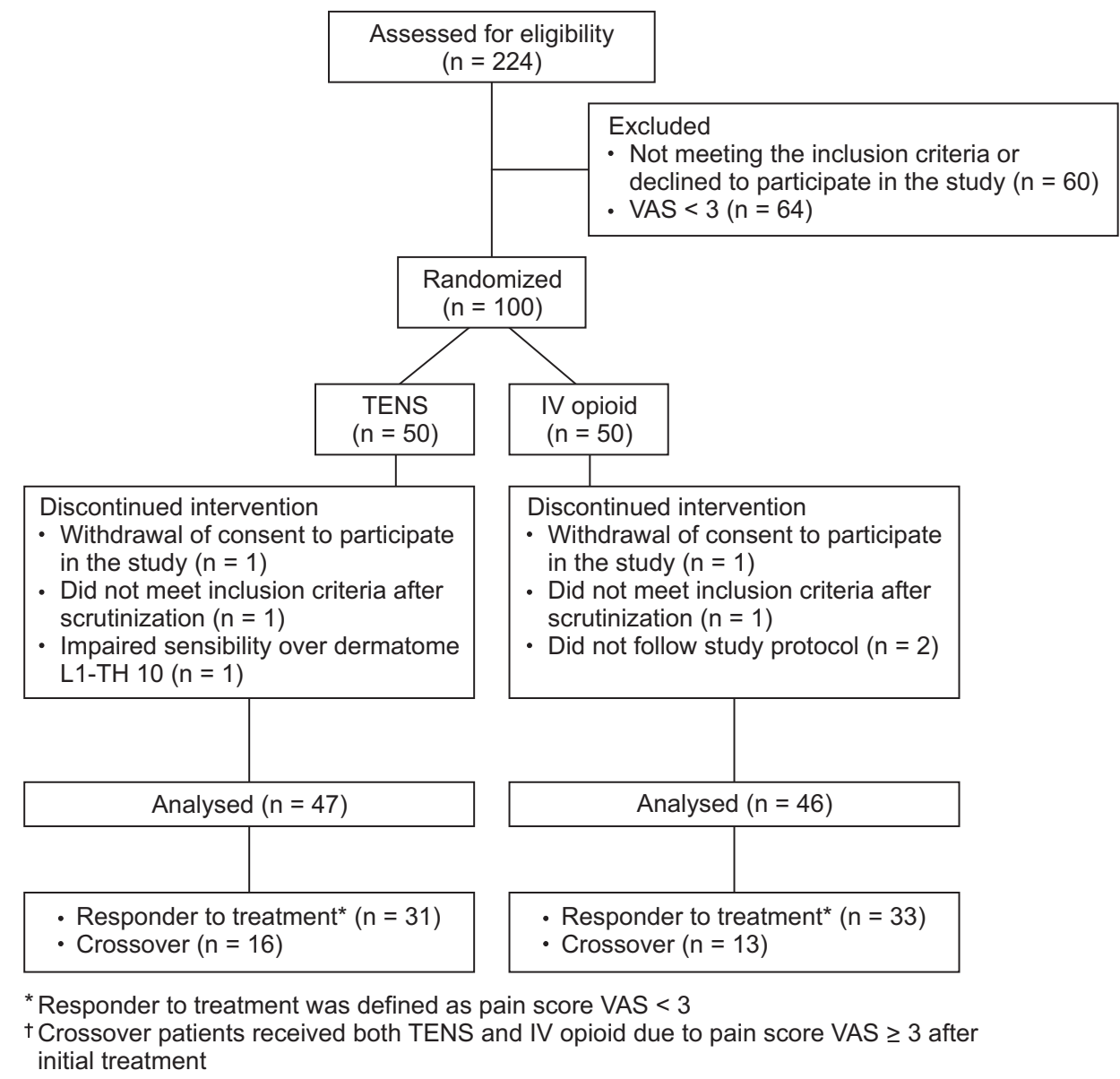

Fig. 1. Patient flowchart. VAS: visual analogue scale, TENS: transcutaneous electrical nerve stimulation, IV: intravenous.

cations. During surgery, the patients received propofol-remifentanil for general anesthesia. The majority of the patients $(n=84)$ were administered a standard dose $(10 \mathrm{mg})$ of IV morphine, 9 patients were administered IV morphine as needed. The laparoscopic procedure was performed according to standard clinical procedure specific for the indicated surgery.

\section{Postoperative procedures}

In the TENS group, the electrodes were placed over the area of pain, usually at dermatome Th11-L1 (Fig. 2) with high-frequency $(80 \mathrm{~Hz})$ stimulation $\left(\right.$ CefarPrimo ${ }^{\circledR}$, DJO France S.A.S Center, France) for approximately $60 \mathrm{~s}$. Since all the patients received local infiltration in the skin during surgery, a cold test was performed in order to determine skin sensibility in the recovery unit. Intact skin sensibility is a prerequisite for TENS treatment. High-intensity stimulation was achieved through increasing the stimulation step by step to reach a stimulation intensity of 40 to $60 \mathrm{~mA}$. The pain intensity was re-assessed immediately after the end of treatment, i.e., after $60 \mathrm{~s}$ of stimulation. The procedure

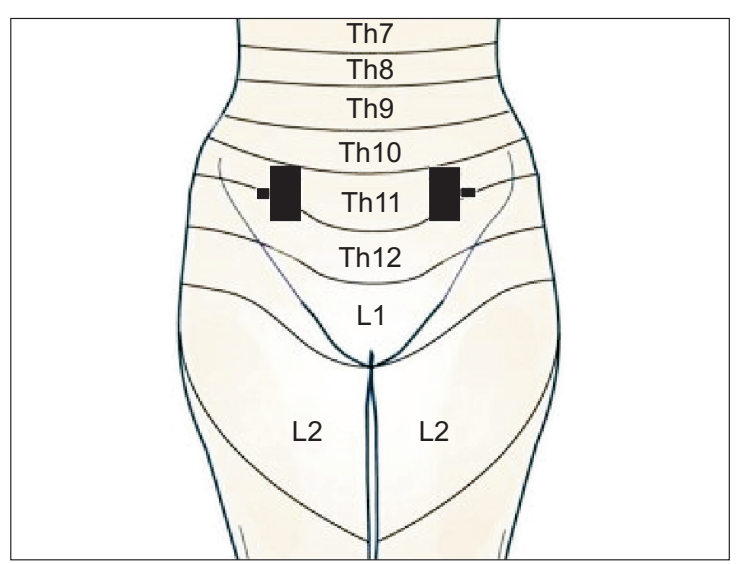

Fig. 2. Electrode placement Th11-L1 when using transcutaneous electrical nerve stimulation (TENS) for postoperative pain relief after gynecologic laparoscopic surgery.

was repeated once, if needed to achieve a VAS score $<3$. If pain relief was not obtained after two sessions of TENS stimulation, the patient received IV opioids. If the patient reported that the 
Table 2. Premedication and Pharmacologic Treatments during the Perioperative Period in the TENS and IV Opioid Treatment Groups

\begin{tabular}{lcc}
\hline & $\begin{array}{c}\text { TENS group } \\
(\mathrm{n}=47)\end{array}$ & $\begin{array}{c}\text { IV opioid group } \\
(\mathrm{n}=46)\end{array}$ \\
\hline $\begin{array}{l}\text { Premedication } \\
\text { NSAID }\end{array}$ & $44(94 \%)$ & $39(85 \%)$ \\
Paracetamol & $47(100 \%)$ & $46(100 \%)$ \\
Pharmacological treatment during surgery & \\
Total intravenous anesthesia & $47(100 \%)$ & $45(98 \%)$ \\
Bupivacaine infiltration sc & $47(100 \%)$ & $45(98 \%)$ \\
Fentanyl IV & $0(0 \%)$ & $2(4 \%)$ \\
Postoperative pharmacological treatment & \\
Morphine IV & $16(34 \%)$ & $46(100 \%)$ \\
Tramadol IV* & $4(9 \%)$ & $2(4 \%)$ \\
Other analgesics in the recovery & $1(2 \%)$ & $2(4 \%)$ \\
unit* & & \\
\hline
\end{tabular}

Data are presented as number of patients (\%). All patients received IV morphine during surgery. TENS: transcutaneous electrical nerve stimulation, IV: intravenous, NSAID: non-steroidal anti-inflammatory drugs, sc: subcutaneous, SD: standard deviation. *Only crossover patients received additional analgesics, including tramadol, postoperatively.

stimulation was unbearable, the patient received additional IV opioid after the first session.

In the IV opioids group, a fractionated dose of morphine was given. The pain intensity was re-estimated after approximately $10 \mathrm{~min}$ after injection. If the patient reported insufficient pain relief, which was determined by a VAS score $>3$ after approximately $10 \mathrm{~min}$, extra doses of IV morphine were administered, until a total dose of $10 \mathrm{mg}$ IV. If the patient still reported inadequate pain relief after $30 \mathrm{~min}$ from the start of pain treatment, TENS was administered. The non-responders to treatment in both groups received adjuvant analgesics according to the local clinical guidelines (Table 2).

A nurse assessed all patients for nausea and sedation in the recovery unit. Nausea was assessed using VAS (0-10), and sedation was estimated by the Ramsay sedation score [13]. The patients were transferred to the unit when their cardiorespiratory status was considered stable (according to standard clinical criteria) and adequate pain relief had been achieved (VAS $<3$ ). The times of the patient's arrival in the recovery unit and discharge from the unit were recorded in the patient's chart.

\section{Statistical analysis}

For the comparison between the 2 groups, Fisher's exact test was used for dichotomous variables. Chi-squared analysis was used for non-ordered categorical variables. The Mann-Whitney $U$ test was used for continuous variables. For the comparison within the groups, Wilcoxon signed rank test was used for continuous variables. All tests were two-tailed, and a significance level of $5 \%$ was used.
Sample size was calculated for a power of $80 \%$ with time spent in the recovery unit as the primary outcome variable. In order to demonstrate a difference between the groups with $80 \%$ power and a two-sided significance level of alpha 0.05, a sample size of 50 participants in each group (time at the recovery unit in minutes, mean 44.3 (30.7) vs. 62.1 (34.3) in the TENS and IV opioid group, respectively) was required [12]. The data were analyzed based on an intention-to-treat (ITT) analysis. A subgroup analysis was performed based on 'responders to treatment.'

\section{Results}

\section{Patients}

In total, 224 patients were assessed for eligibility after admission to the operating theater at Sahlgrenska University Hospital/ Östra for gynecologic laparoscopic surgery. Sixty-four patients declined to participate in the study and 60 patients did not meet the inclusion criteria (pain score was not $\geq 3$ on the VAS). In total, 100 patients had pain requiring treatment (VAS pain score $\geq 3$ ) and were randomized to receive TENS $(n=50)$ or standard pharmacological treatment with IV opioids $(n=50)$; however, 2 of the patients did not meet all of the inclusion criteria after scrutiny, 2 patients withdrew their consent for study participation, 1 patient had impaired sensibility, and the study protocol was not followed for 2 patients (Fig. 1).

Between the two groups, there were no significant differences in their health characteristics (Table 1), use of premedication, pharmacological treatment during surgery, and or postoperatively pharmacological treatment (Table 2). The patients who underwent gynecologic laparoscopic procedures are presented in Table 1. Mean time of surgery was 68.7 (32.7) min in the TENS group and 58.3 (33.2) $\mathrm{min}$ in the IV opioid group. The opioid group was administered a fractionated dose of morphine as needed for pain relief [mean $7.7(3.0) \mathrm{mg}$ ]. In the TENS group, 7 patients did not accept the high-intensity stimulation. For these cases, a level of 20-30 mA was reached, and one of the patients crossed over and received an IV opioid.

There were no significant differences between the groups with regard to the number of crossovers (patients who reported VAS $\geq 3$ after treatment). Thirteen patients in the opioid group received additional TENS treatment and 16 patients in TENS group received additional IV opioids $(\mathrm{P}=0.66)$. Of the crossover patients, one patient in the TENS group received IV fentanyl and IV esketamine in addition to IV morphine. One patient in the opioid group received IV oxycodone due to allergies against morphine and one patient received IV esketamine in addition to TENS treatment (Table 2). 
Table 3. Pain Intensity, Pain Relief, and Duration of Hospital Stay in the TENS Treatment Group and in the IV Opioid Treatment Group (Intention-to-treat Analysis)

\begin{tabular}{lccc}
\hline & $\begin{array}{c}\text { TENS group } \\
(\mathrm{n}=47)\end{array}$ & $\begin{array}{c}\text { IV opioid group } \\
(\mathrm{n}=46)\end{array}$ & P value \\
\hline $\begin{array}{l}\text { VAS before treatment } \\
\begin{array}{l}\text { VAS upon discharge from } \\
\text { the recovery unit* }\end{array}\end{array}$ & $5.4(1.6)$ & $5.2(1.7)$ & 0.505 \\
$\begin{array}{c}\text { Duration of stay in the } \\
\text { recovery unit (min) }\end{array}$ & $110.5(48.7)$ & $1.1(1.1)$ & 0.801 \\
$\begin{array}{c}\text { VAS at hospital discharge } \\
\text { Duration of post-surgical } \\
\text { hospital stay (min) }\end{array}$ & $1.5(1.1)$ & $2.4(1.9)$ & 0.037 \\
\hline
\end{tabular}

Data are presented as mean (SD) and median. TENS: transcutaneous electrical nerve stimulation, IV: intravenous, VAS: visual analogue scale, ${ }^{*} \mathrm{P}$ values within groups; TENS $<0.001$, IV opioid $<0.001$. ${ }^{\dagger}$ TENS $\mathrm{n}=$ 39 (missing $n=8$ ), IV opioid $n=33$ (missing $n=13$ ). ${ }^{*}$ Duration of postsurgical hospital stay and stay in the recovery unit; TENS $n=47$, IV opioid $n=43$.

\section{Duration of stay in the recovery unit and pain relief}

According to the ITT analysis, there were no significant differences in the duration of stay in the recovery unit between the groups (TENS group: $110.5 \mathrm{~min}$; IV opioid group: $120.1 \mathrm{~min}$; $\mathrm{P}=0.44$ ) or the duration of hospital stay after surgery (TENS group: $393.5 \mathrm{~min}$; IV opioid group: $452.6 \mathrm{~min}$; $\mathrm{P}=0.30$ ). However, when only responders to the treatment (i.e., patients who reported VAS $<3$ on assigned treatment) were compared (per protocol analysis), the TENS responders had a significantly shorter stay in the recovery unit when compared to that of the responders in the opioid group (Table 4).

Both groups reported significant pain relief (TENS group: VAS 5.4 to $1.0, \mathrm{P}<0.001$; IV opioid group: VAS 5.2 to $1.1, \mathrm{P}<$ 0.001 ) (Table 3). More than one third of the patients reported complete pain relief, without any differences between the groups (TENS group: $\mathrm{n}=17$ [36.2\%]; IV opioid group: $\mathrm{n}=15$ [32.6\%]; $\mathrm{P}=0.83$ ). When only responders to treatment were compared, approximately half of the patients in the TENS group reported complete pain relief (TENS group: $\mathrm{n}=15$ [48.4\%]; IV opioid group: $\mathrm{n}=12$ [36.4\%]; $\mathrm{P}=0.45$ ). There were no significant differences between the groups with regard to pain after surgery or pain relief upon discharge from the recovery unit. Upon discharge from the hospital, the TENS group reported significantly less pain than the IV opioid group (TENS group: VAS 1.5: IV opioid group: VAS 2.4; $\mathrm{P}=0.037$ ) (Table 3 ).

In total, 31 patients $(66.0 \%)$ in the TENS group and 33 patients $(71.7 \%)$ in the IV opioid group reported VAS $<3$ after the initial treatment of pain. Patients who did not respond to their assigned treatment (i.e., crossover patients [ $n=29,31.2 \%]$ ) had a higher VAS before (6.2 vs. 4.9 ) and after treatment (1.4 vs. 0.8 ) when compared to that of the responders to treatment. Cross-
Table 4. Pain Intensity, Pain Relief, and Duration of Hospital Stay in the Patients Who Responded to TENS Treatment and IV Opioids (Per Protocol Analysis)

\begin{tabular}{|c|c|c|c|}
\hline & $\begin{array}{l}\text { TENS group } \\
\quad(\mathrm{n}=47)\end{array}$ & $\begin{array}{l}\text { IV opioid group } \\
\quad(\mathrm{n}=46)\end{array}$ & $P$ value \\
\hline VAS before treatment & $4.9(1.6)$ & $4.9(1.2)$ & 1.000 \\
\hline $\begin{array}{l}\text { VAS upon discharge from } \\
\text { recovery unit }\end{array}$ & $0.7(0.7)$ & $1.0(1.0)$ & 0.229 \\
\hline $\begin{array}{l}\text { Duration of stay in the } \\
\text { recovery unit (min) }\end{array}$ & $89.8(36.3)$ & $122.2(60.0)$ & 0.008 \\
\hline $\begin{array}{l}\text { VAS upon hospital } \\
\text { discharge* }\end{array}$ & $1.2(1.1)$ & $2.4(2.0)$ & 0.020 \\
\hline $\begin{array}{l}\text { Duration of post-surgical } \\
\text { hospital stay }(\mathrm{min})^{\dagger}\end{array}$ & $337.0(289.4)$ & $477.7(639.2)$ & 0.512 \\
\hline
\end{tabular}

Data are presented as mean (SD). TENS: transcutaneous electrical nerve stimulation, IV: intravenous, VAS: visual analogue scale. ${ }^{*}$ TENS $n=28$ (missing $\mathrm{n}=3$ ); IV opioid $\mathrm{n}=25$ (missing $\mathrm{n}=8$ ). ${ }^{\dagger}$ Duration of postsurgical hospital stay and stay in the recovery unit; TENS $n=31$, IV opioid $n=31$ (missing $n=2)$.

over patients also stayed in the recovery unit for a longer period than the responders to treatment ( 134.7 vs. $106.5 \mathrm{~min}$ ); there were more patients with long-term pain in this group $(62.1 \%$ vs. $25.0 \%, \mathrm{P}=0.001$ ).

\section{Nausea and sedation}

There were no significant differences with regard to sedation, nausea, or use of anti-emetics between the groups.

\section{Discussion}

The current randomized study demonstrated that treatment with high-intensity, high-frequency stimulation TENS, as well as conventional treatment with IV opioids, are effective for postoperative pain relief after gynecologic laparoscopic surgery. More than one third of the patients reported complete pain relief. This is consistent with the results from previous studies, indicating that TENS is effective for postoperative pain relief after laparoscopic surgery $[7,8]$. However, there were no differences between the groups with regard to the primary endpoint (i.e., duration of stay in the recovery unit).

According to clinical practice guidelines, patients should be monitored for $30 \mathrm{~min}$ after the last morphine injection due to the risk of respiratory depression. In contrast, there is no need to monitor patients after TENS treatment. When only responders to treatment were compared, both groups reported similar pain relief, but the TENS group had a shorter stay in the recovery unit. If a patient responded to TENS treatment, the patient reported a prompt onset of the pain relief, and monitoring for adverse side effects such as sedation, nausea, and respiratory depression was not necessary. Since it takes only $2 \mathrm{~min}$ to evaluate 
for a patient's response to TENS, it seems to be the preferable first choice for treatment of postoperative pain.

In contrast, it takes approximately $30 \mathrm{~min}$ to evaluate the effect of IV opioid treatment [14]. Hence, initial pain treatment with TENS does not delay IV opioid treatment by more than a few minutes if the patients do not respond to TENS treatment.

There were 29 crossovers (31.2\%) in the study, with no differences between the groups. Since it is more difficult to achieve adequate postoperative pain relief in patients with long-term pain, a possible explanation might be the high percentage of patients with long-term pain in the crossover group (Table 1) [15]. Furthermore, TENS may have had an inferior effect in some patients treated with opioids prior to surgery [16].

It is well known that opioids have several side effects, such as severe respiratory depression and sedation. Postoperative nausea is a less severe side effect, although it is time- and drug-consuming and is unpleasant for the patient. Since all patients received general anesthesia and IV morphine during surgery, this might be a reason why there were no differences between the groups with regard to postoperative nausea.

\section{Clinical implication}

In the current study, high-frequency $(80 \mathrm{~Hz})$, high-intensity (40 to $60 \mathrm{~mA}$ ) TENS stimulation for $60 \mathrm{~s}$ was used and, if needed, repeated once. This is the same treatment model used for primary dysmenorrhea, angina pectoris, and surgical abortion $[12,17,18]$.

The TENS device delivers electrical impulses through the skin using sticky pad electrodes placed on the lower abdomen. This causes paresthesia over the area of the abdominal pain and alleviates post-surgical pain. For optimum pain relief, adjusting the position of the electrodes so that the paresthesia covers the area to which the patient localizes the pain seems to be an important measure. Before the start of the treatment, skin sensibility should be assessed, since TENS should not be used if there is impaired sensibility.

Some patients reported an uncomfortable stimulation during the 1 min of the initial stimulation with TENS as a side effect. The stimulation lasted only $60 \mathrm{~s}$, and all the women in the TENS group were informed that this short period of (uncomfortable) high-intensity TENS was needed in order to obtain postoperative pain relief. To ease the patients, the stimulation intensity was increased step by step in order to achieve a high-intensity level. Aside from the discomfort, TENS has no other known acute side effects, and there are no reports of side effects, such as sedation and respiratory depression. Skin irritation is another reported side effect of long-term TENS treatment, which was not observed in the current study, most likely due to the short treatment sessions. TENS stimulation also causes disturbances in electrocardiogram (ECG) readings, which has previously been described [12].

When the TENS device is turned off, the ECG monitor is not affected. Since the patients are stimulated for only a short period of time, this disturbance of the ECG is not considered harmful or clinically significant. In addition, the patients are generally healthy young women, and the ECG monitoring is mainly used for assessing the respiratory rate. This approach for postoperative pain treatment require regular education and training of the staff, as well as easily accessible TENS devices and electrodes.

\section{Limitations of the study}

First, as a clinical routine in our institution, patients received paracetamol and NSAID during their hospital stay and IV morphine during surgery for preventive analgesia. However, only patients reporting pain after surgery in the recovery unit were included in the study and hence received treatment for pain.

Second, the indications for undergoing gynecologic laparoscopic surgery differed between the groups. However, there was no difference with regard to postoperative pain according to the VAS scores in the recovery unit prior to the initiation of treatment for pain between the IV opioid and TENS groups. Hence, the indication for surgery does not seem to affect the postoperative pain reported by the patients.

Third, for ethical reasons, it was not possible to have a control group that did not receive treatment of postoperative pain. Hence, TENS was compared to IV opioids, which is considered 'the gold standard' for postoperative pain relief.

Fourth, it would have been a great advantage to be able to blind the patients and the investigator to treatment allocation. However, due to technical and clinical logistical reasons, it was not possible to blind the investigators, since it was impossible to blind the patients to their treatment allocation results. This might have affected the outcome. Nevertheless, it should be emphasized that the patients reported their pain intensity using the VAS (i.e., the nurse did not estimate or influence the patient's report).

In addition, approximately one third of the patients in both groups needed further pain relief and thus crossed over to the other treatment arm, indicating that both interventions were insufficient for pain control in these patients. The large number of crossovers might have affected the results.

Fifth, for the crossover patients, we cannot rule out a possible carry-over effect of the TENS and the IV opioid treatment that might have influenced the results [19]. The results from per-protocol analyses indicate that both treatments, IV opioids and TENS, were effective, but the initial treatment with TENS results in a shorter duration of stay in the recovery unit if the patient responds to the treatment. However, the data should be 
interpreted with caution since the 'responder to treatment analysis' is a subgroup analysis and not the primary ITT analysis.

Sixth, the TENS group reported less pain upon discharge from the hospital. However, the data should be interpreted cautiously due to the large number of missing data in both groups.

\section{Conclusions}

Both TENS and short-term IV opioids are effective treatments for pain after gynecologic laparoscopic surgery, with more than one third of the patients reporting complete pain relief. There were no differences between the groups with regard to duration of stay in the recovery unit. However, since it is associated with a shorter duration of stay in the recovery unit if the patient responds to the treatment, TENS seems to be preferable as the first line treatmentIn addition, the effect of treatment can be assessed quickly and does not delay IV opioid treatment if the patients do not respond to TENS treatment. However, more than $30 \%$ of the patients required further pain relief; any single intervention seems to be insufficient for all patients. Further randomized controlled trials are needed in order to confirm the results of this study.

\section{Acknowledgments}

We would like to thank our medical statisticians, Professor Anders Odén and Nils-Gunnar Pehrsson, for their expert professional advice. We would also like to thank Associate Professor Sven-Egron Thörn for his support and expert scientific advice. Finally, we acknowledge the support of patients and the nurses at the recovery unit and the gynecologic unit whose work made this study possible.

\section{ORCID}

Birgitta Platon, https://orcid.org/0000-0002-9992-9578

Clas Mannheimer, https://orcid.org/0000-0003-3233-0568

Paulin Andréll, https://orcid.org/0000-0001-6941-6096

\section{References}

1. Fehintola AO, Awotunde OT, Ogunlaja AO, Olujide LO, Akinola SE, Oladeji SA, et al. Preliminary report of experience with gynaecologic laparoscopy in Ogbomoso, Nigeria. Gynecol Surg 2016; 13: 17-21.

2. Alexander JI. Pain after laparoscopy. Br J Anaesth 1997; 79: 369-78.

3. Borgbjerg FM, Nielsen K, Franks J. Experimental pain stimulates respiration and attenuates morphine-induced respiratory depression: a controlled study in human volunteers. Pain 1996; 64: 123-8.

4. Freynet A, Falcoz PE. Is transcutaneous electrical nerve stimulation effective in relieving postoperative pain after thoracotomy? Interact Cardiovasc Thorac Surg 2010; 10: 283-8.

5. Kara B, Baskurt F, Acar S, Karadibak D, Ciftci L, Erbayraktar S, et al. The effect of TENS on pain, function, depression, and analgesic consumption in the early postoperative period with spinal surgery patients. Turk Neurosurg 2011; 21: 618-24.

6. Tokuda M, Tabira K, Masuda T, Nishiwada T, Shomoto K. Effect of modulated-frequency and modulated-intensity transcutaneous electrical nerve stimulation after abdominal surgery: a randomized controlled trial. Clin J Pain 2014; 30: 565-70.

7. Desantana JM, Sluka KA, Lauretti GR. High and low frequency TENS reduce postoperative pain intensity after laparoscopic tubal ligation: a randomized controlled trial. Clin J Pain 2009; 25: 12-9.

8. Silva MB, de Melo PR, de Oliveira NM, Crema E, Fernandes LF. Analgesic effect of transcutaneous electrical nerve stimulation after laparoscopic cholecystectomy. Am J Phys Med Rehabil 2012; 91: 652-7.

9. Bjerså K, Andersson T. High frequency TENS as a complement for pain relief in postoperative transition from epidural to general analgesia after pancreatic resection. Complement Ther Clin Pract 2014; 20: 5-10.

10. Bjerså K, Jildenstaal P, Jakobsson J, Egardt M, Fagevik Olsén M. Adjunct high frequency transcutaneous electric stimulation (TENS) for postoperative pain management during weaning from epidural analgesia following colon surgery: results from a controlled pilot study. Pain Manag Nurs 2015; 16: 944-50.

11. Moran F, Leonard T, Hawthorne S, Hughes CM, McCrum-Gardner E, Johnson MI, et al. Hypoalgesia in response to transcutaneous electrical nerve stimulation (TENS) depends on stimulation intensity. J Pain 2011; 12: 929-35.

12. Platon B, Andréll P, Raner C, Rudolph M, Dvoretsky A, Mannheimer C. High-frequency, high-intensity transcutaneous electrical nerve stimulation as treatment of pain after surgical abortion. Pain 2010; 148: 114-9.

13. van Dishoeck AM, van der Hooft T, Simoons ML, van der Ent M, Scholte op Reimer WJ. Reliable assessment of sedation level in routine clinical practice by adding an instruction to the Ramsay Scale. Eur J Cardiovasc Nurs 2009; 8: 125-8.

14. Aubrun F, Mazoit JX, Riou B. Postoperative intravenous morphine titration. Br J Anaesth 2012; 108: 193-201.

15. Erlenwein J, Przemeck M, Degenhart A, Budde S, Falla D, Quintel M, et al. The influence of chronic pain on postoperative pain and function after hip surgery: a prospective observational cohort study. J Pain 2016; 17: 236-47. 
16. Solomon RA, Viernstein MC, Long DM. Reduction of postoperative pain and narcotic use by transcutaneous electrical nerve stimulation. Surgery 1980; 87: 142-6.

17. Mannheimer C, Carlsson CA, Vedin A, Wilhelmsson C. Transcutaneous electrical nerve stimulation (TENS) in angina pectoris. Pain 1986; 26: 291-300.

18. Milsom I, Hedner N, Mannheimer C. A comparative study of the effect of high-intensity transcutaneous nerve stimulation and oral naproxen on intrauterine pressure and menstrual pain in patients with primary dysmenorrhea. Am J Obstet Gynecol 1994; 170: 123-9.

19. Murray S, Collins PD, James MA. An investigation into the 'carry over' effect of neurostimulation in the treatment of angina pectoris. Int J Clin Pract 2004; 58: 669-74. 